\title{
Enhancement of VEGF on Axial Vascularization of Nano-HA/Collagen/PLA Composites: A Histomorphometric Study on Rabbits
}

\author{
Xiao Chang, ${ }^{1}$ Hai Wang, ${ }^{1}$ Zhihong Wu, ${ }^{1}$ Xiaojie Lian, ${ }^{2}$ Fuzhai Cui, ${ }^{2}$ Xisheng Weng, \\ Bo Yang, ${ }^{1}$ Guixing Qiu, ${ }^{1}$ and Baozhong Zhang ${ }^{1}$ \\ ${ }^{1}$ Department of Orthopaedic Surgery, Peking Union Medical College Hospital, No. 1, Shuaifuyuan Hutong, Beijing 100730, China \\ ${ }^{2}$ Department of Materials Science \& Engineering, Tsinghua University, Beijing 100084, China
}

Correspondence should be addressed to Baozhong Zhang; zbz9639@sina.com

Received 28 January 2014; Accepted 6 March 2014; Published 31 March 2014

Academic Editor: Xiaoming Li

Copyright (C) 2014 Xiao Chang et al. This is an open access article distributed under the Creative Commons Attribution License, which permits unrestricted use, distribution, and reproduction in any medium, provided the original work is properly cited.

The aim of this study was to investigate whether the nanohydroxyapatite/collagen/poly(L-lactic acid) (nHAC/PLA) composite is suitable to be compounded with VEGF to enhance the axial vascularization in vivo. Thirty rabbits were divided into 2 groups of 15 animals each. In control group, a nHAC/PLA scaffold slice was vascularized axially by an inserted ligated femoral arteriovenous (AV) bundle in the animal. In experimental group, a slice compounded with VEGF gel was applied. The rabbits were sacrificed at 2 weeks, 6 weeks, and 10 weeks after surgery; the specimens of scaffold slices underwent histomorphometric examination; analysis of the microvessel density (MVD) of both groups was done. The combination with VEGF (Group B) did not enhance the vascularization in early phase $(2$ and 6 weeks, $P>0.05)$ but worked in later phase $(10$ weeks, $P<0.05)$. The data of the experiment demonstrated the suitability of the nHAC/PLA composite as carrier for the growth factor VEGF, enabling its sustained release in bioactive form with enough binding efficacy.

\section{Introduction}

Critical-sized bone defect due to serious trauma, infection, malignant tumor resection, or congenital deformity is not rare in clinic and remains a major challenge for surgeons. Tissue engineered bone (TEB) represents a promising method to resolve these problems. In the future, TEB might become the most popular choice for surgeons when they repair segmental bone defects [1]. Significant progress has been made to develop several bone substitute materials which mimics natural bone matrix in both composition and microstructure [2-4]. Some kinds of seed cells and bioactive factors have been composited with scaffolds by many tissue engineering methods [5]. However, repair of bone defects over $30 \mathrm{~mm}$ using TEB still is a difficult problem [6], because seed cells and bioactive factors will survive only with oxygen and nutrition supplied by blood flow in limited scale. The osteoinduction and osteogenesis of large tissue-engineered scaffold will not function because of absence of vascularization in the scaffold.

Vascularization plays a very important role in skeletal development and repair [7]. To repair large bone defect, it is critical to vascularize the whole scaffold; there is more new bone formation where the matrix is vascularized more adequately [8]. There are two methods to promote the angiogenesis of matrix of TEM. First, through microsurgical procedure, a major vessel is implanted in the scaffold to enhance angiogenesis around the vessel which is called axial vascularization. Second, angiogenic factors are composited with the scaffold to induce vessels formation. In the factors the vascular endothelial growth factor (VEGF) and bone morphogenetic protein (BMP) are the most commonly used [9].

Nanohydroxyapatite/collagen/poly(L-lactic acid) (nHAC/ PLA) composite is a new type bone substitute scaffold; it is similar to the natural bone in main composition and in 
hierarchical microstructure [9-12]. In previous studies we have revealed that the $\mathrm{nHAC/PLA}$ composites can be axial vascularized by femoral arteriovenous bundle of rabbits [13]. The purpose of this study was to investigate whether VEGF can be loaded on nHAC/PLA composites to promote the axial vascularization of the scaffold in vivo.

\section{Materials and Methods}

2.1. Materials. Process of synthesis of nHAC/PLA has been previously described [9-13]. After ultrasonication, the material was fabricated into cylindrical slices $(\Phi 10 \mathrm{~mm} \times 10 \mathrm{~mm})$ with a hole of $1.5 \mathrm{~mm}$ diameter in the center.

2.2. Experimental Design. The whole experiment was approved by the Animal Ethic Committee of Peking Union Medical College Hospital (PUMCH), and the procedures were conducted in accordance with the guidelines for the care and maintenance of animals. Thirty-three-month-old New Zealand rabbits (Experimental Animal Center of PUMCH, Beijing, China) weighing 2.5 to $3.0 \mathrm{~kg}$ were divided randomly into group A and group B $(n=15)$. All operations were performed under sterile conditions by one surgeon. In group A (control group), a nHAC/PLA composite slice was directly implanted into the intramuscular gap with an arteriovenous (AV) bundle in the left groin of a rabbit. In group B (experimental group), the $\mathrm{nHAC/PLA}$ composite slice was coated with VEGF165 gel and then implanted into the animal with the same procedure as group A. Histological examinations were performed at 2, 6, and 10 weeks after implantation.

\subsection{Experimental Steps}

2.3.1. Compositing of VEGF. In clean bench, 10 cylindrical slices were dripped into $50 \mathrm{~mL}$ fibrinogen solution at $50 \mathrm{mg} / \mathrm{mL}$; the same volume of VEGF165 solution at $5 \mathrm{ng} / \mathrm{mL}$ was added in. Then the mixture of VEGF165 and fibrinogen infiltrated into the micropores of nHAC/PLA through vacuum suction for 30 minutes. The $100 \mathrm{~mL}$ thrombin solution at $250 \mathrm{U} / \mathrm{ml}$ was added in and the vacuum suction was repeated for 30 minutes. The thrombin transferred fibrinogen to gel which contained VEGF165. After being removed from the solution and air-dried, the slices were kept in sterile and $4^{\circ} \mathrm{C}$ environment until operation.

2.3.2. Surgical Protocol. With an intravenous injection of $3 \%$ pentobarbital sodium ( $1 \mathrm{~mL} / \mathrm{kg}$ body weight, Sigma, USA), the animals were anesthetized. Before procedures, 800,000 IU penicillin sodium (North China Pharmaceutical Group Corporation, China) was injected intramuscularly in order to prevent perioperative infection. The animals were placed on the table in supine position. After shaving, sterilizing, and draping, through a skin incision from the left groin midpoint to the left knee, the femoral neurovascular bundle was exposed, and the nerve was protected. The left femoral artery and vein were surgically dissociated and then were transversely cut and ligated at 2 to $3 \mathrm{~cm}$ under the femoral artery furcation for an AV bundle. In group A, the AV bundle

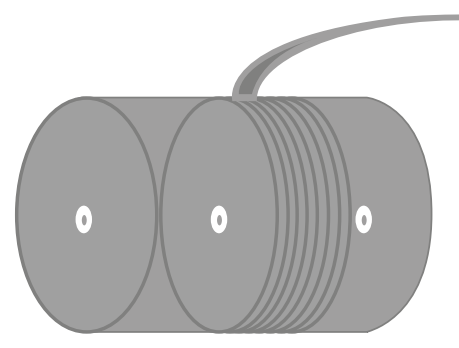

(a)

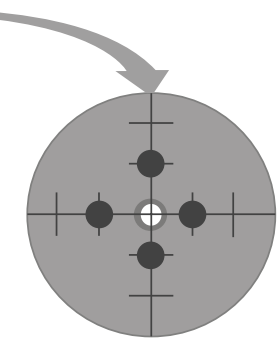

(b)
FIGURE 1: Stick drawing demonstrating the section process of histological examinations: (a) 8 cross-sections were obtained perpendicular to the AV bundle in the middle; (b) 4 microphotographs of interest in the inner $1 / 3$ radius at $3,6,9$, and 12 oclock at $400 \mathrm{x}$ magnification were evaluated for MVD (dark black points) [13].

was placed in the hole of a nHAC/PLA composite slice, and the slice was placed into the intramuscular gap. In group $B$, the nHAC/PLA composite slice coated with VEGF165 gel was applied to place AV bundle in. Femoral muscle and skin were sutured with 3-0 silk sutures. Postoperatively, 800,000 IU penicillin sodium and $0.15 \mathrm{mg}$ buprenorphine (Tianjing Institute of Pharmaceutical Research, China) were separately administered intramuscularly every 12 hours for 3 days.

2.4. Histological Examinations. The animals were sacrificed after 2,6 , and 10 weeks ( $n=5$, resp.). The slices were removed and fixed in $10 \%$ buffered formalin for 24 hours. After washing, they were decalcified by $14 \%$ ethylenediaminetetraacetic acid (EDTA) solution for 4-6 weeks. Then, they were dehydrated in graded ethanol and embedded in paraffin. Eight cross-sections $(5 \mu \mathrm{m})$ were obtained from each specimen, perpendicular to the AV bundle in the middle (Figure 1(a)), using a Leica microtome (Leica Microsystems, Wetzlar, Germany). For histomorphometric analysis, four sections were randomly selected for haematoxylin and eosin (H\&E) staining and microphotographs were taken using a microscope and a digital camera (Leica Microsystems); see Figure 5. On each section, four microphotographs of interest in the inner $1 / 3$ radius at $3,6,9$, and 12 o'clock at $400 x$ magnification were evaluated, and the number of vessels in high power field (HPF) was counted by two independent and blinded pathologists (Figure 1(b)). Microvessel density (MVD) was calculated for each group and each time point. One of the other sections was chosen randomly for CD31 immunohistochemical staining for qualitative assessment.

2.5. Statistics Analysis. The MVDs were given as means \pm standard deviation ( $\mathrm{X} \pm \mathrm{SD}$, vessels/HPF). Statistical analysis was performed using the paired samples Student's $t$-test with SPSS 16.0. The critical level of statistical significance was set at $P<0.05$.

\section{Result}

3.1. Surgery and Macroscopic Appearance. 27 rabbits tolerated the surgical procedure and survived well. One rabbit in group 
TABLE 1: Quantification of MVD (X $\pm \mathrm{SD}$, vessels/HPE) between group A and group $\mathrm{B}$, analyzed by histomorphometrics. $P$ values $<0.05$ were marked $(*)$.

\begin{tabular}{lccc}
\hline & Group A (controlled group without VEGF) & Group B (experimental group with VEGF) & $P$ value \\
\hline 2 weeks & $9.35 \pm 1.58$ & $9.02 \pm 1.60$ & 0.897 \\
6 weeks & $15.60 \pm 3.20$ & $16.04 \pm 2.89$ & 0.917 \\
10 weeks & $17.35 \pm 2.64$ & $20.92 \pm 3.91$ & $0.048^{*}$ \\
\hline
\end{tabular}

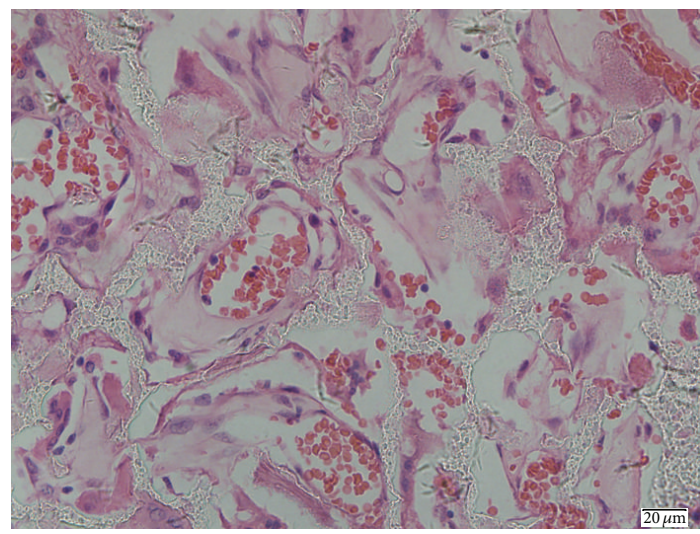

Figure 2: Hematoxylin and eosin (HE) staining of specimens 10 weeks after implantation (magnification $\times 400$ ) showed the tube architecture of the newly formed vessels.

A and one in group B died of diarrhea in two weeks after procedures; one in group A was complicated with incision infection. Above-mentioned three animals were excluded from the experiment and 3 new animals were selected to replace the excluded. No extrusion of the implanted slices occurred over the observation period. The slices were surrounded by neighboring tissue when removal was performed; vessel-like tissues were observed on the surface of the implanted slices of both groups.

3.2. Histological Examinations. Microscopic angiogenesis was identified in both groups of any time point. At low magnification, the vision was full of connective tissue and granulation tissue composed of inflammatory cells, blood cells, fibroblasts, and blood vessels; at high magnification, vascular endothelial cells could be identified (Figure 2). Around the ligation AV bundle there were new vessels formed which indicated arteriogenesis (Figure 3). The new vessels formed in the slices could be identified through CD31 immunohistochemical staining; the endothelial cells of vessel wall showed significant light brown (Figure 4). Along with the time extension, there were fewer inflammatory cells and more fibroblasts and vessels. By 10 weeks, more vessels even arteriole-like ones were observed in both groups.

A trend towards an increase in MVD was observed in each group over time, and MVD of group B gradually showed its significance more than that of group A. At 2 and 6 weeks after implantation, MVD of groups A and B had no significant differences $(P=0.897$ and $P=0.917)$; at the last time point of 10 weeks, the MVD of group B was significant more than group A $(P=0.048)$ (Table 1$)$.

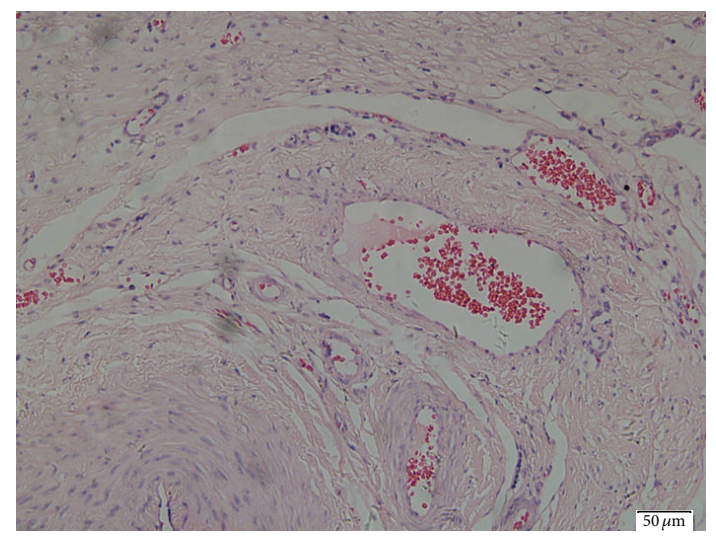

FIgURE 3: HE staining of specimens 10 weeks after implantation (magnification $\times 200)$ showed arteriogenesis: new collateral vessels formed around ligation AV bundle.

\section{Discussion}

Angiogenesis is a prerequisite for bone formation. The newly formed vessel net supplies the site of bone formation with nutrients, oxygen, and soluble factors [14]. Vascularization also seems particularly to be important for the healing process following the implantation of bone substitute materials $[1,15]$. The establishment of a dense vascular network is essential for bone formation, osseointegration, and the subsequent material replacement by newly formed bone [16]

In previous studies, we have established animal models for axial vascularization of the nHAC/PLA composite and improved the scaffold to be applicable to axial vascularization of vessel bundle [13].

The distal ligation arteriovenous (AV) bundle is one of axial vascular carriers. Compared with the other two methods, shunt loop and flow-through type AV bundle, the distal ligation AV bundle is easy to construct and can be used as the pedicle vessels if transposition repair is necessary. The distal ligation AV bundle has been used clinically as vascular carriers $[17,18]$. The vascularization of AV bundle is based on arteriogenesis. Upon occlusion of an artery, the blood flow is redirected into preexisting arteriolar anastomoses that experience increased mechanical forces such as shear stress and circumferential wall stress. The endothelium of the arteriolar connections is then activated, resulting in an increased release of monocyte-attracting proteins as well as an upregulation of adhesion molecules [19]. Upon adherence and extravasation, monocytes promote arteriogenesis by supplying growth factors and cytokines that bind to receptors that are expressed on vascular cells within a limited 


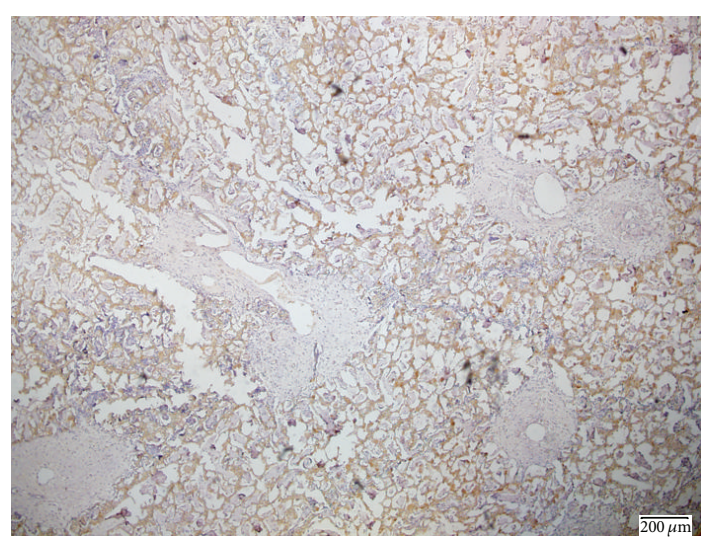

(a)

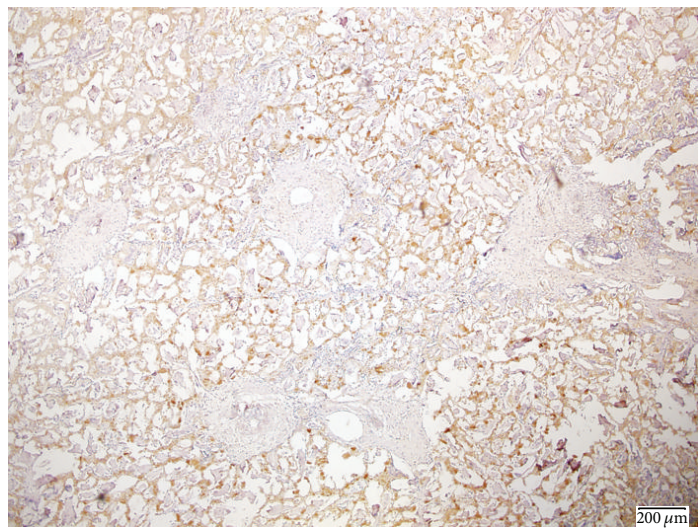

(b)

FIGURE 4: CD31 immunohistochemical staining of specimens 10 weeks after implantation (magnification $\times 20$ ): the arteriole-like vessels can be seen, (a) from group A, (b) from group B.

time frame [20]. There is ischemic zone around AV bundle secondary to surgical trauma and ligation of vessels. The ischemia and hypoxia cause new collateral vessels formation through cell proliferation and vessel budding [21].

The results of histological examinations showed good biocompatibility and property for angiogenesis of nHAC/PLA composite. In early period of implantation, there was inflammatory cells in the scaffold which indicated foreign body reaction; in late period, the connective tissue replaced the inflammatory tissue and new vessels formed. The count of microvessels showed that the AV bundle promotes the vascularization of the scaffold. Though it is difficult to repair critical-sized bone defect for the nHAC/PLA composite, it is a promising scaffold material to construct TEB to repair larger bone defects in the future [22].

To upload the bioactive factors with the scaffold is another method to promote vascularization of the scaffold and VEGF is used most commonly. More recently, VEGF has gained increasing attention because it has been demonstrated to be mostly capable of stimulating angiogenesis in bone grafts. VEGF is highly specific for vascular endothelial cells and its bioactivity in angiogenesis is stronger than any of the other

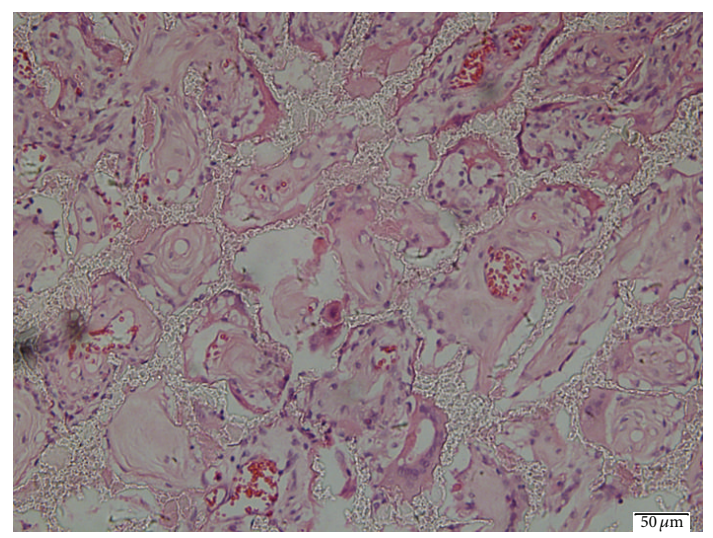

(a)

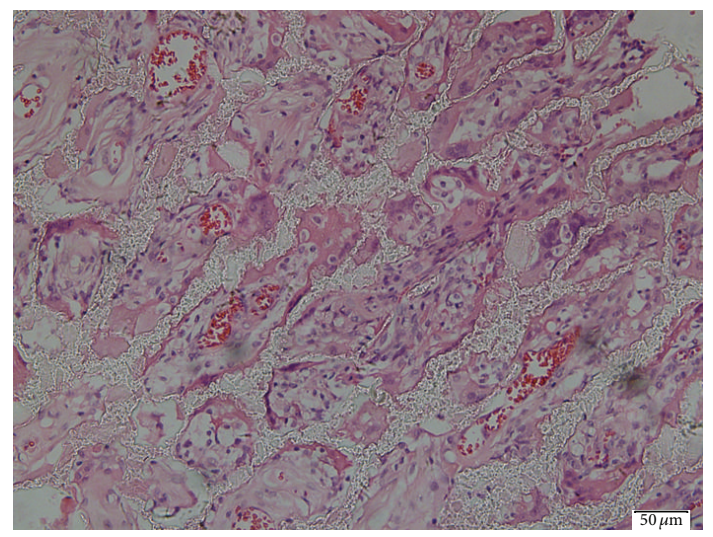

(b)

FIGURE 5: HE staining of specimens (magnification $\times 100$ ): the vascular walls and red blood cells can be seen in both groups 10 weeks after implantation: (a) from group A; (b) from group B.

known proangiogenic factors; any proangiogenic factor promotes vessel formation through VEGF directly or indirectly. In vivo, VEGF induces angiogenesis and permeabilization of blood vessels. VEGF165 is the most active member of the family; by binding receptors in the endothelial cells, it can stimulate endothelial cells to divide and proliferate; it also can be the chemokine to push the EPCs to migrate from bone marrow to get involved in angiogenesis [23].

In the experiment, VEGF165 was loaded on the scaffold and released slowly in animals. Because of the short half-life in vivo, VEGF165 degrades into inactive fragments quickly. To prevent it from degradation, different methods were applied to keep the activity of VEGF165 and release the activity gradually $[24,25]$. The method in the study was similar to that introduced by Lode A $[26,27]$. The mixed solution of VEGF165 and fibrinogen adhered to the scaffold by vacuum suction, and the mixture transferred to gel when thrombin was added in. The activity of VEGF165 was kept in the gel and released to function slowly.

The effect of angiogenesis by VEGF released from the nHAC/PLA composite was not significant until 10 weeks after implantation. In early time points, the histological examination showed new vessel formation in the scaffolds of 
both groups, but there was no significant difference in MVDs by statistic analysis. It was most likely due to lack of cells and tissues as the targets of VEGF in early time after implantation. With time, especially when inflammatory reaction was over, the pores of scaffold were full of living tissue, and there were enough target-cells of VEGF to activate. As a result, the MVD of the experimental group was significantly higher than that of the controlled group in 10 weeks.

There are still problems to resolve for VEGF in promotion of angiogenesis of the substitute bone materials. The safety of VEGF in vivo is unknown because VEGF is closely related to the development and progression of tumors. The procedure of compounding VEGF and the scaffold is the lack of standardization. The concentration and release speed vary due to different microstructure of the scaffolds. There still is a lot of research work to do to bridge the laboratory experimentation to practical clinical settings.

\section{Conclusions}

The data of the experiment demonstrated the suitability of the nHAC/PLA composite as carrier for the growth factor VEGF, enabling its sustained release in bioactive form with enough binding efficacy. The microstructure of this kind of scaffold not only fits new born tissue growing after axial vascularization, but also fits coating of growth factor such as VEGF. The nHAC/PLA composite is proved to be a promising scaffold material to construct the TEB in the future.

\section{Conflict of Interests}

The authors declare that there is no conflict of interests regarding the publication of this paper.

\section{Acknowledgment}

This paper is sponsored by the National Natural Science Foundation of China under Contract Grant no. 81000802. Xiao Chang and Hai Wang are co-first authors.

\section{References}

[1] X. Li, L. Wang, Y. Fan, Q. Feng, F. Z. Cui, and F. Watari, "Nanostructured scaffolds for bone tissue engineering.," Journal of Biomedical Materials Research A, vol. 101, no. 8, pp. 24242435, 2013.

[2] X. Li, C. A. van Blitterswijk, Q. Feng, F. Cui, and F. Watari, “The effect of calcium phosphate microstructure on bone-related cells in vitro," Biomaterials, vol. 29, no. 23, pp. 3306-3316, 2008.

[3] G. Zhou, Y. Hou, L. Liu et al., "Preparation and characterization of NiW-nHA composite catalyst for hydrocracking," Nanoscale, vol. 4, no. 24, pp. 7698-7703., 2012.

[4] G. Zhou, Z. Wu, Y. Hou et al., "Research on the structure of fish collagen nanofibers influenced cell growth," Journal of Nanomaterials, vol. 2013, Article ID 764239, 6 pages, 2013.

[5] X. Li, H. Liu, X. Niu et al., "The use of carbon nanotubes to induce osteogenic differentiation of human adipose-derived MSCs in vitro and ectopic bone formation in vivo," Biomaterials, vol. 33, no. 19, pp. 4818-4827, 2012.
[6] X. Li, Q. Feng, X. Liu, W. Dong, and F. Cui, "Collagen-based implants reinforced by chitin fibres in a goat shank bone defect model," Biomaterials, vol. 27, no. 9, pp. 1917-1923, 2006.

[7] L. L. Ren, D. Y. Ma, X. Feng, T. Q. Mao, Y. P. Liu, and Y. Ding, "A novel strategy for prefabrication of large and axially vascularized tissue engineered bone by using an arteriovenous loop," Medical Hypotheses, vol. 71, no. 5, pp. 737-740, 2008.

[8] J. DELEU and J. TRUETA, "Vascularisation of bone grafts in the anterior chamber of the eye," The Journal of Bone and Joint Surgery, vol. 47, pp. 319-329, 1965.

[9] J. Li, J. Hong, Q. Zheng et al., "Repair of rat cranial bone defects with nHAC/PLLA and BMP-2-related peptide or rhBMP-2," Journal of Orthopaedic Research, vol. 29, no. 11, pp. 1745-1752, 2011.

[10] S. S. Liao, F. Z. Cui, W. Zhang, and Q. L. Feng, "Hierarchically biomimetic bone scaffold materials: nano-HA/collagen/PLA composite," Journal of Biomedical Materials Research B: Applied Biomaterials, vol. 69, no. 2, pp. 158-165, 2004.

[11] C. Du, F. Z. Cui, W. Zhang, Q. L. Feng, X. D. Zhu, and K. de Groot, "Formation of calcium phosphate/collagen composites through mineralization of collagen matrix," Journal of Biomedical Materials Research, vol. 50, no. 4, pp. 518-527, 2000.

[12] C. Du, F. Z. Cui, X. D. Zhu, and K. de Groot, "Threedimensional nano-HAp/collagen matrix loading with osteogenic cells in organ culture," Journal of Biomedical Materials Research, vol. 44, no. 4, pp. 407-415, 1999.

[13] H. Wang, X. Chang, G. Qiu et al., "Axial vascularization of Nano-HA/Collagen/PLA composites by arteriovenous bundle," Journal of Nanomaterials, vol. 2013, Article ID 391832, 6 pages, 2013.

[14] J. Harper and M. Klagsbrun, "Cartilage to bone-angiogenesis leads the way," Nature Medicine, vol. 5, no. 6, pp. 617-618, 1999.

[15] J. M. Kanczler and R. O. C. Oreffo, "Osteogenesis and angiogenesis: the potential for engineering bone," European Cells and Materials, vol. 15, pp. 100-114, 2008.

[16] E. Wernike, M.-O. Montjovent, Y. Liu et al., "Vegf incorporated into calcium phosphate ceramics promotes vascularisation and bone formation in vivo," European Cells and Materials, vol. 19, pp. 30-40, 2010.

[17] J. J. Pribaz, P. K. M. Maitz, and N. A. Fine, "Flap prefabrication using the "vascular crane" principle: an experimental study and clinical application," British Journal of Plastic Surgery, vol. 47, no. 4, pp. 250-256, 1994.

[18] K. C. Tark, R. K. Khouri, K. S. Shin, and W. W. Shaw, "The fasciovascular pedicle for revascularization of other tissues," Annals of Plastic Surgery, vol. 26, no. 2, pp. 149-155, 1991.

[19] E. Deindl and W. Schaper, "The art of arteriogenesis," Cell Biochemistry and Biophysics, vol. 43, no. 1, pp. 1-15, 2005.

[20] K. Kosaki, J. Ando, R. Korenaga, T. Kurokawa, and A. Kamiya, "Fluid shear stress increases the production of granulocytemacrophage colony-stimulating factor by endothelial cells via mRNA stabilization," Circulation Research, vol. 82, no. 7, pp. 794-802, 1998.

[21] A. Arkudas, G. Pryymachuk, J. P. Beier et al., "Combination of extrinsic and intrinsic pathways significantly accelerates axial vascularization of bioartificial tissues," Plastic and Reconstructive Surgery, vol. 129, no. 1, pp. 55e-65e, 2012.

[22] X. ] Li, Y. Yang, Y. Fan, Q. Feng, F. Z. Cui, and F. Watari, "Biocomposites reinforced by fibers or tubes as scaffolds for tissue engineering or regenerative medicine," Journal of Biomedical Materials Research A, 2013. 
[23] E. Boscolo, J. B. Mulliken, and J. Bischoff, "VEGFR-1 mediates endothelial differentiation and formation of blood vessels in a murine model of infantile hemangioma," The American Journal of Pathology, vol. 179, no. 5, pp. 2266-2277, 2011.

[24] Q. Tan, H. Tang, J. Hu et al., "Controlled release of chitosan/heparin nanoparticle-delivered VEGF enhances regeneration of decellularized tissue-engineered scaffolds," International journal of nanomedicine, vol. 6, pp. 929-942, 2011.

[25] P. Yang, C. Wang, Z. Shi et al., "Prefabrication of vascularized porous three-dimensional scaffold induced from rhVEGF165: a preliminary study in rats," Cells Tissues Organs, vol. 189, no. 5, pp. 327-337, 2009.

[26] A. Lode, A. Reinstorf, A. Bernhardt, C. Wolf-Brandstetter, U. König, and M. Gelinsky, "Heparin modification of calcium phosphate bone cements for VEGF functionalization," Journal of Biomedical Materials Research A, vol. 86, no. 3, pp. 749-759, 2008.

[27] A. Lode, C. Wolf-Brandstetter, A. Reinstorf et al., "Calcium phosphate bone cements, functionalized with VEGF: release kinetics and biological activity," Journal of Biomedical Materials Research A, vol. 81, no. 2, pp. 474-483, 2007. 

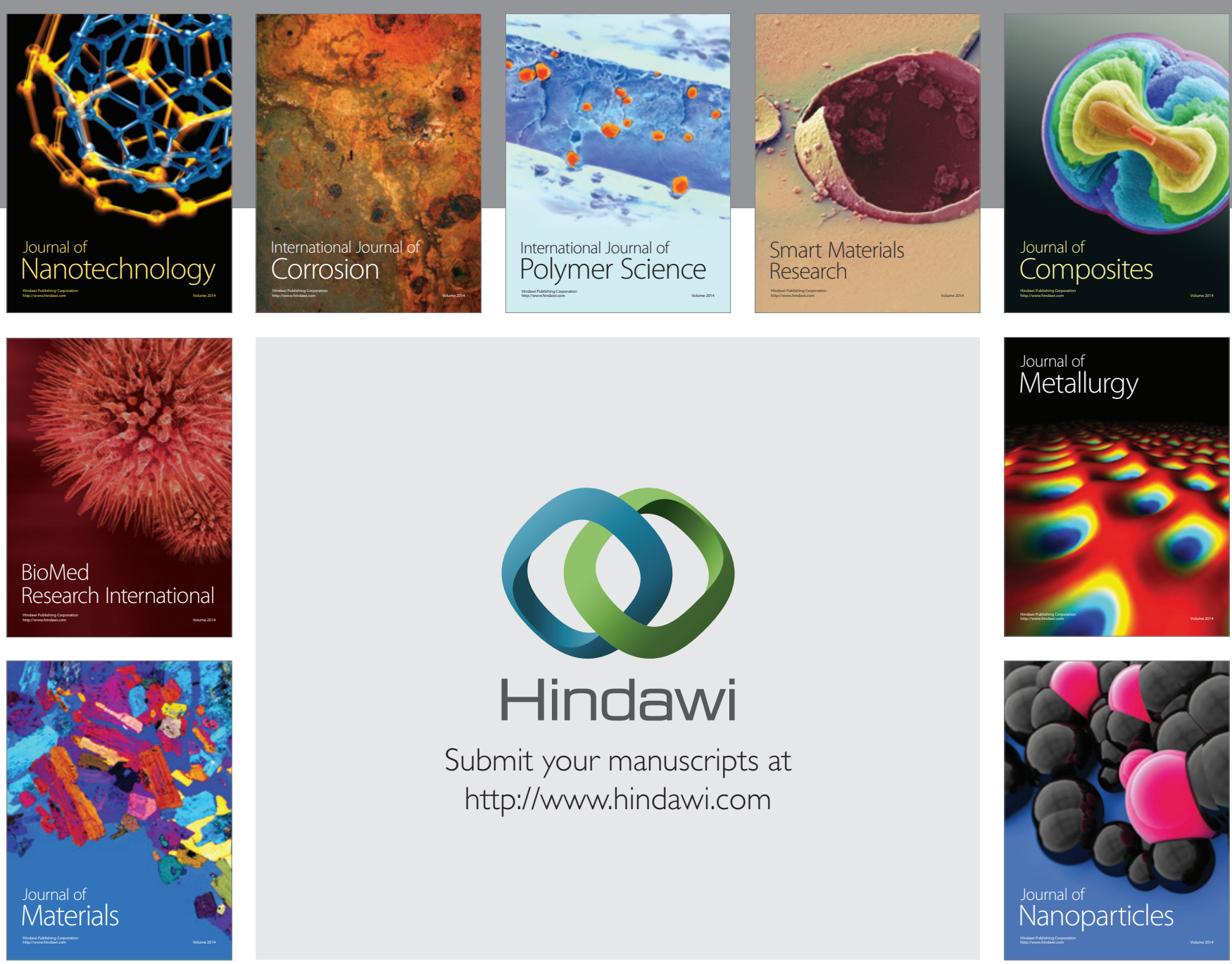

Submit your manuscripts at http://www.hindawi.com
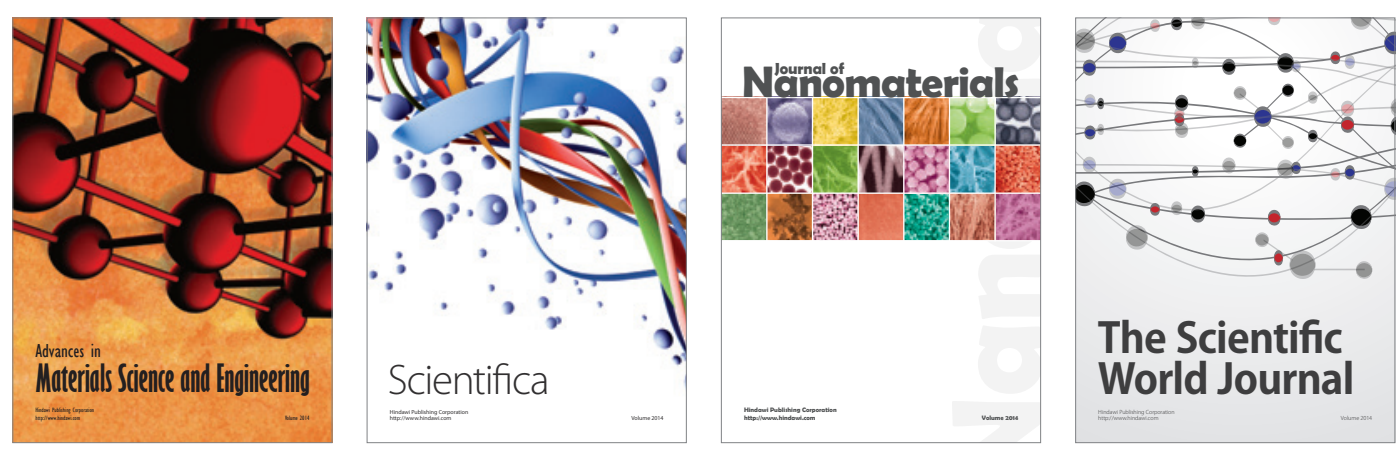

\section{The Scientific World Journal}
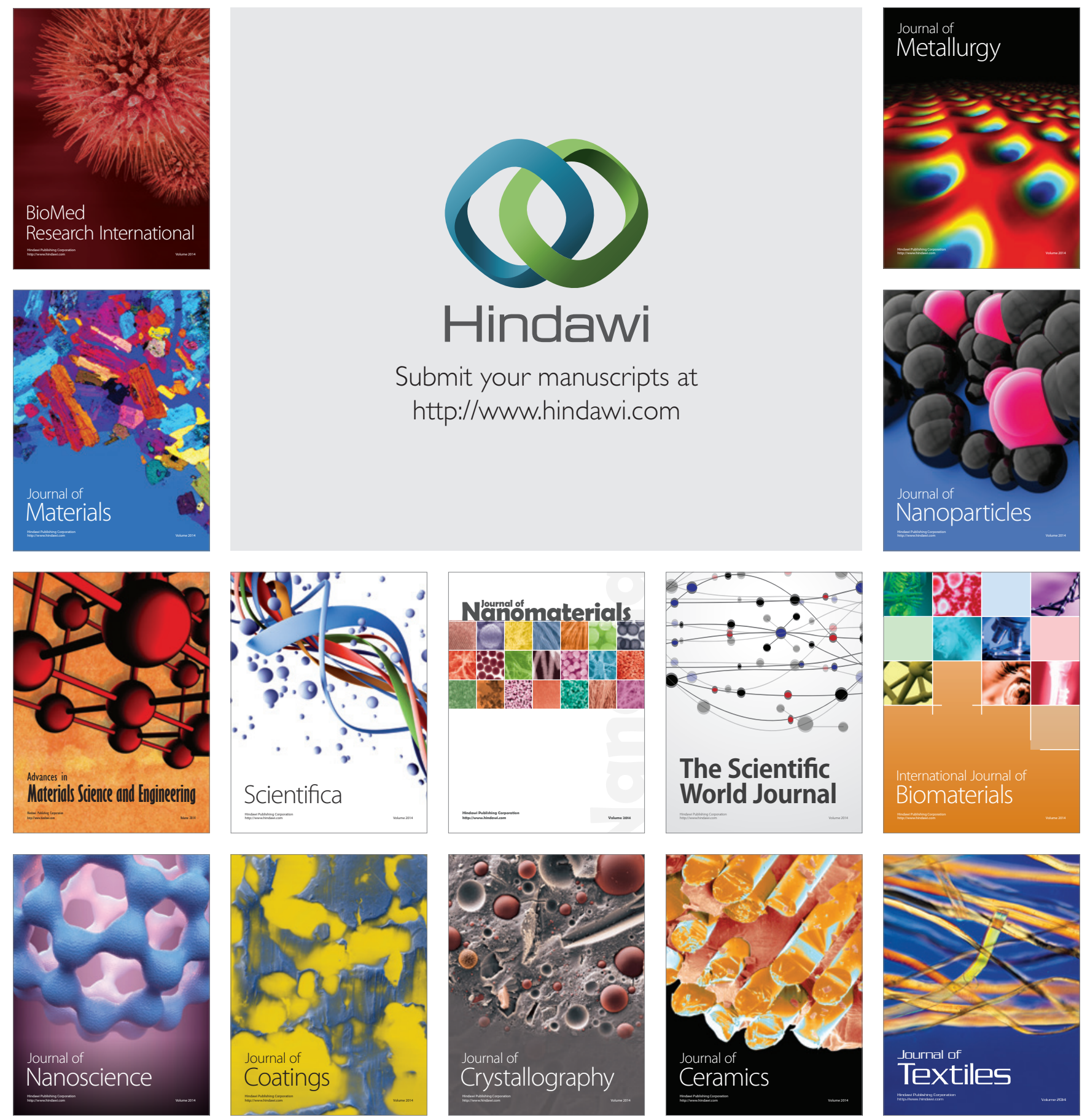